This item was submitted to Loughborough's Research Repository by the author.

Items in Figshare are protected by copyright, with all rights reserved, unless otherwise indicated.

\title{
Material model for modeling clay at high strain rates
}

PLEASE CITE THE PUBLISHED VERSION

http://dx.doi.org/10.1016/j.ijimpeng.2015.11.005

\section{PUBLISHER}

(c) Elsevier

VERSION

AM (Accepted Manuscript)

\section{PUBLISHER STATEMENT}

This work is made available according to the conditions of the Creative Commons Attribution-NonCommercialNoDerivatives 4.0 International (CC BY-NC-ND 4.0) licence. Full details of this licence are available at: https://creativecommons.org/licenses/by-nc-nd/4.0/

\section{LICENCE}

CC BY-NC-ND 4.0

\section{REPOSITORY RECORD}

Buchely, M.F., Alejandro Maranon, and Vadim V. Silberschmidt. 2019. "Material Model for Modeling Clay at High Strain Rates". figshare. https://hdl.handle.net/2134/20070. 


\title{
Material model for modeling clay at high strain rates
}

\author{
M.F. Buchely ${ }^{\mathrm{a}}$, A. Maranon ${ }^{\text {a,* }}$, V.V. Silberschmidt ${ }^{\mathrm{b}}$ \\ a Structural Integrity Research Group, Mechanical Engineering Department, Universidad de los Andes, CR 1 ESTE 19A 40, Bogota 111711, Colombia
} ${ }^{b}$ Woifson School of Mechanical and Manufacturing Engineering, Loughborough University, Loughtborough, Leics LE11 3 TU UK

\begin{abstract}
A B S T R A C T
Modeling clay is a soft malleable material made from oils and waxes. This material is fundamental for ballistic evaluation of body armors because it is used as backing material in ballistic tests. After a ballistic impact, a back-face indentation is measured to assess performance of the armor. Due to the important role of modeling clay in this particular application, its mechanical characterization and comprehension of penetration mechanics are essential for development of new personal protection systems. This paper presents a two-step computational methodology to calibrate parameters of a Cowper-Symonds material model for modeling clay at characteristic strain rates up to $1.8 \times 10^{4} \mathrm{~s}^{-1}$. In the first stage, a highspeed camera is used to record the penetration of a gas-gun launched cylindrical mass with a hemispherical cap into a block of clay. Image-processing software is used to capture the tail of the projectile as it penetrates into the clay. These data are then used to sample the penetration depth as function of time. In the second stage, an in-house developed model of penetration, based on both the spherical cavity expansion theory and the Tate penetration equation, is used to determine, by inverse analysis, the parameter of the Cowper-Symonds clay model. The proposed constitutive relationship for clay and the determined material parameters can be applied accurately to problems involving high strain rates.
\end{abstract}

\section{Introduction}

Modeling clay is a thixotropic material (a material which has the property of being time-dependent shear thinning) made from inert mineral oils, fillers and waxes [1], which is commonly used as a recording medium of back-face deformation of soft and hard body armors in back-face signature (BFS) tests [2]. Simple modeling clay, such as Roma Plastilina No.1, is used in blunt trauma research, and in many countries, it is a standard backing material for bodyarmor testing since it provides some benefits in relatively lowvelocity impacts compared to other backing materials [3]. Hence, comprehension of the mechanical response and penetration mechanics of modeling clay are fundamental for analysis of ballistic body-armor tests.

Despite the fact that modeling clay has been used for this purpose for nearly 25 years [4], there are no available material models that describe its behavior at the strain rates characteristic to such tests. Some approximations to its behavior are only limited to low strain rates. Chijiiwa et al. [5] performed a quasi-static and dynamic characterization of plasticine at strain-rates up to $10 \mathrm{~s}^{1}$, using conventional compression tests and drop weight tests. The behavior

- Corresponding author. Structural Integrity Research Group, Mechanica Engineering Department, Universidad de los Andes, CR 1 ESTE 19A 40, Bogota 111711, Colombia. Tel.: +5713324322; Fax: +5713324323

E-mail address: emaranon@uniandes.edu.co (A. Maranon). of plasticine was adjusted to the Ludwik constitutive relation. Sofuoglu and Rasty $[6,7]$ performed quasi-static $\left(10^{-3} \mathrm{~s}^{-1}\right)$ compression tests to determine the flow behavior of various types of plasticine. The effect of strain was only considered, modeling plas ticine using the strain dependent power-law equation. Huang et al. [8] employed an analytical model of a spherical and conical indentation test to determine the behavior of plasticine, which was described by the Herschel-Bulkley relationship. In this research, effective strain-rates during the test were not calculated. However the indentation was performed at low penetration velocities $(0.1-4 \mathrm{~mm} / \mathrm{s})$. Eckerson et al. [9] conducted a thermomechanical characterization of plasticine using compression tests at different compression velocities $\left(10^{-2} \mathrm{~s}^{-1}\right.$ to $\left.10 \mathrm{~s}^{-1}\right)$. In this case, the NortonHoff viscoplastic model was used to approximate the response of plasticine. More recently, Hernandez et al. [10] performed dynamic characterization of modeling clay by drop tests at medium strain rates $\left(10^{2} s^{-1}\right)$, adjusting plasticine behavior to the Ludwik constitutive relation.

At higher strain rates, the behavior of the modeling clay has been slightly described. Crandall et al. [11,12] measured the Poisson's ratio and the elastic modulus of plasticine clay using resonance tests at frequencies up to $1000 \mathrm{~Hz}$. Some researchers have approximated the plasticine behavior at high-strain rates using experimental data and empirical relations (e.g., Munusamy and Barton [13] and Park et al. [3]). However, those relations cannot be extrapolated. Consequently, there is no sufficient clarity about the mechanical behavior of modeling clay at high strain rates. This is a significant limitation 
for body-armor designers as they cannot evaluate the BFS of a given concept using finite-element explicit computational codes.

One of the difficulties for modeling clay is its thixotropic be havior [14], where its viscosity depends on time, strain rate and temperature [1,3]. An additional difficulty is the lack of a standard test method to characterize this kind of soft materials due to its low elastic modulus, which impedes the use of contact devices for measuring its dynamic mechanical properties [15].

In this paper, a two-step methodology is presented to calibrate parameters of a Cowper-Symonds material model [16] for modeling clay at high strain rates (according to the classification given by Nemat-Nasser [17]). First, a technique to characterize soft materials at high strain rates was designed based on the Dynamic Indentation test and high-speed images. The test was performed at constant temperature to avoid the temperature sensitivity of the modeling clay. Second, an engineering model of penetration, based on both the spherical cavity expansion theory $[18,19]$ and the Tate penetration equation $[20,21]$, was formulated. The material parameters were calibrated by an inverse technique employing the experimental data and the engineering model. Finally, these parameters, and the ones found by traditional quasi-static uni-axial compression tests, were used in numerical finite -element (FE) models in order to compare their solutions with experimental data.

\section{Theoretical background}

\subsection{Penetration regimes}

According to Backman and Goldsmith [22], the phenomenology of the target in a penetration event can be divided in fou regimes depending on the penetration velocity $\left(V_{p}\right)$ : (1) elastic de formations in the target at extremely low $V_{p}$, where the target is deformed but recovers its shape; (2) plastic deformation, when $V_{p}$ is high enough to achieve pressures on the impact surface above the compression yield stress of the material $\left(\sigma_{y}\right)$, and the target ex periences a variety of phenomena including propagation of elastic, plastic, and/or hydrodynamic waves as well as frictional heating that produces local and global deformations; (3) a hydrodynamic regime, when $\sqrt{\sigma_{y} / \rho}<V_{p}<\sqrt{K / \rho}$ (where $\rho$ is the material density and $K$ is the bulk modulus), and the material can be modeled as a fluid; anc (4) hyper-velocity impacts, at sufficiently high impact velocities, demonstrating decrease of compressibility of the solid, shock waves, comminution, phase changes, vaporization, or even impact explosions of the target material.

Most of the penetration models are focused on the second and third regimes since they involve medium and high penetration ve locities $[20,21]$. However, special care should be taken with soft materials due to their low bulk modulus, as they can easily progress into the fourth penetration regime.

\subsection{Cowper-Symonds plasticity material model}

The plastic flow behavior of the material should be reflected in a constitutive model. The Cowper-Symonds (CS) plasticity constitutive model [16] is typically used for super-plastic forming analysis due to its dependency on plastic strain $\left(\varepsilon_{\mathrm{pl}}\right)$ and a plastic strain rate $\left(\dot{\varepsilon}_{\text {मे }}\right)$ :

$\sigma_{e}=\left(Y_{0}+B \varepsilon_{p l}^{n}\right)\left(1+\left(\frac{\dot{\varepsilon}_{p l}}{D}\right)^{Y q}\right)$.

In this model, $\sigma_{e}$ is the effective flow stress, $Y_{0}$ is the yield stress at zero plastic strain, $B$ is the strain-hardening coefficient, $n$ is the strain-hardening exponent, and $D$ and $q$ are strain-rate-hardening parameters.
According to Boutelier et al. [23], viscous materials, as modeling clay, are considered as strain independent, like a Reiner-Rivlin fluid [24]. It means that the state of stress depends only on the velocity gradient. Therefore, a simplified version of the CowperSymonds model (equation 2) can be used to describe the behavior of modeling clay at high strain-rates.

$\sigma_{e}=Y_{0}\left(1+\left(\frac{\dot{\varepsilon}_{p l}}{D}\right)^{V_{q}}\right)$.

\section{Spherical cavity-expansion theory}

Spherical cavity-expansion theory has been commonly used to calculate the pressure on the cavity wall during a penetration process. It is based on the spherical deformation of the field, where spherical symmetry is used to reduce the formulation.

In a previous paper [25], the problem of the spherical cavity expansion in a compressible, elastic-viscoplastic Cowper-Symonds field was solved. The system was numerically solved; and after solution, the cavity pressure was found, and it was used to model penetration problems. In the current formulation, the spherical cavity expansion problem was reduced, assuming modeling clay as an incompressible material, both in the elastic and the plastic branch. In the elastic branch, plasticine is incompressible due to its high Poissons ratio $(v=0.43)[11,12]$. In the plastic branch, Green [26] posed that plastic compressibility in plasticine is negligible if all air bubbles are removed by working the material. This was corroborated by Feng et al. [27] using SEM images of deformed plasticine specimens. As incompressibility is assumed, the system can be analytically solved.

Using this considerations (spherical symmetry and incompressibility), mass- and momentum-conservation equations in spherical coordinates were reduced to the following one-dimensional problem [28]:

$\dot{\varepsilon}_{\tau}+2 \dot{\varepsilon}_{\ominus}=0$,

$\frac{\partial \sigma_{r}}{\partial r}+2 \frac{\sigma_{r}-\sigma_{\vartheta}}{r}=\rho \dot{v}$,

where $\mathrm{r}$ is a Eulerian radial coordinate, $\dot{\varepsilon}_{\tau}=\partial v / \partial \mathrm{r}$ is the radial strain rate; $\dot{\varepsilon}_{\theta}=v / r$ is the hoop strain rate; $v=\dot{r}$ is the radial material velocity (it was taken positive outward); $\rho$ is the material density; and $\sigma_{7}, \sigma_{\theta}, \sigma_{\phi}$ are the radial, hoop and meridional components of Cauchy stress tensor taken positive in compression, respectively, and $\sigma_{\theta}=\sigma_{\phi}$ from symmetry [29].

The material response is modeled as a J2 Mises solid [30], where effective stress $\sigma_{e}$ is given by:

$\sigma_{e}=\sigma_{\theta}-\sigma_{r}$.

Assuming the Mises flow theory in the absence of material spin [30-32], the strain-rates and material velocity are related by:

$\dot{\varepsilon}_{r}=\frac{\dot{\sigma}_{r}}{E}-\frac{2 v \dot{\sigma}_{\theta}}{E}-\dot{\varepsilon}_{p l}=\frac{d v}{d r}$

$\dot{\varepsilon}_{\theta}=-\frac{v \dot{\sigma}_{r}}{E}+\frac{(1-v) \dot{\sigma}_{\theta}}{E}+\frac{\dot{\varepsilon}_{p L}}{2}=\frac{v}{r}$

It is assumed that the only independent variable is the nondimensional radial coordinate $\xi=r i a$, where $a$ is the cavity radius (see Fig. 1). Thus, the time derivative is transformed with the similarity relation [32]

$\frac{d}{d t}=\dot{\xi} \frac{d}{d \xi}=\left(\frac{\dot{r}}{a}-\xi \frac{\dot{a}}{a}\right) \frac{d}{d \xi}=\frac{\dot{a}}{a}(V-\xi) \frac{d}{d \xi}$, 


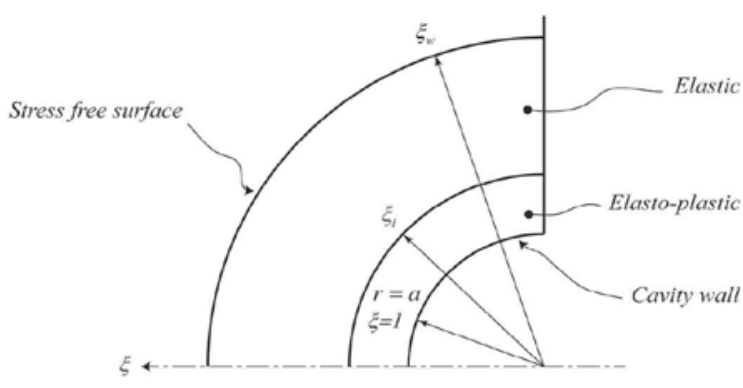

Fig. 1. Schematic representation of material field in spherical cavity expansion.

where $V=\dot{r} / a$ is the nondimensional radial velocity and $\dot{a}$ is the cavity-expansion velocity. Using equation (8), equations (3), (4), (5), (6) and (7) transform to:

$\frac{d V}{d \xi}+2 \frac{V}{\xi}=0$,

$\frac{d \Sigma_{r}}{d \xi}-2 \frac{\Sigma}{\xi}=\dot{A}^{2}(V-\xi) \frac{d V}{d \xi}$,

$\Sigma_{e}=\Sigma_{\theta}-\Sigma_{r}$,

$\frac{d V}{d \xi}=(V-\xi) \frac{d}{d \xi}\left(\Sigma_{r}-2 v \Sigma_{\theta}-\varepsilon_{p l}\right)$,

$\frac{V}{\xi}=(V-\xi) \frac{d}{d \xi}\left(-v \Sigma_{r}+(1-v) \Sigma_{\theta}+\frac{1}{2} \varepsilon_{p l}\right)$,

where $\left(\Sigma, \Sigma_{r}, \Sigma_{\theta}\right)=\left(\sigma_{e}, \sigma_{r}, \sigma_{\theta}\right) / E$ are the non-dimensional stresses, and $\dot{A}$ is the non-dimensional cavity expansion velocity, defined by:

$\dot{A}=\frac{\dot{a}}{\sqrt{E / \rho_{0}}}$.

The mass conservation (equation (9)) has the known solution (using the wall condition $V(\xi=1)=1$ ):

$V=\frac{1}{\xi^{2}}$.

Using this solution (equation (15)), momentum-conservation (equation (10)) is reduced to:

$$
\frac{d \Sigma_{r}}{d \xi}-2 \frac{\Sigma}{\xi}=2 \dot{A}^{2}\left(\frac{\xi^{3}-1}{\xi^{5}}\right),
$$

and the strain-rates - material velocity relations (equations (12) and (13)) are reduced to (using $v=1 / 2$ ):

$\frac{d \Sigma}{d \xi}+\frac{d \varepsilon_{p l}}{d \xi}=\frac{2}{\xi-\xi^{4}}$.

Equations (16) and (17) are the governing equations for the stress components $\left(\Sigma\right.$ and $\left.\Sigma_{r}\right)$ and represent the behavior of the elasto-plastic material. To complete the solution, the cavity mode is divided into two parts: an elastic region, and an elasto-plastic region (Fig. 1). After solution for the last region, pressure for the cavity wall is obtained. Next, the formulation for each region is explained.
2.3.1. Elastic region

In the elastic field $\xi_{i} \leq \xi \leq \infty$, where plastic strain is zero $\left(\varepsilon_{p t} \equiv 0\right)$, solutions of equations (16) and (17), using a stress-free condition as $\xi \rightarrow \infty$, are:

$\Sigma=\frac{2}{3} \ln \left(\frac{\xi^{3}}{\xi^{3}-1}\right)$

$\Sigma_{r}=-\frac{4}{3} \int_{\xi}^{\infty} \ln \left(\frac{x^{3}}{x^{3}-1}\right) \frac{d x}{x}-2 A^{2}\left(\frac{1}{\xi}-\frac{1}{4 \xi^{4}}\right)$.

One approximation for equations (18) and (19) is assumed that as $\xi$ increases, $\xi^{3} \gg 1$ (see reference [28] for more details). Then, the previous equations are reduced to:

$\Sigma=\frac{2}{3 \xi^{3}}$,

$\Sigma_{r}=-\frac{4}{9 \xi^{3}}-2 \dot{A}^{2}\left(\frac{1}{\xi}-\frac{1}{4 \xi^{4}}\right)$

\subsubsection{Elasto-plastic region}

For the elasto-plastic region $1 \leq \xi \leq \xi$, plastic flow can be expressed using the Cowper-Symonds (CS) model (equation (2)). First, it is necessary to find an expression for $\dot{\varepsilon}_{p !}$ in terms of the working variables. Then, using equation (11) and $v=1 / 2$ in equation (6):

$\dot{\varepsilon}_{p l}=-\frac{d v}{d r}-\dot{\Sigma}$.

The behavior of the adjacent plastic layer is dominated by the plastic part of the constitutive relation; then, $\dot{\Sigma}=0$. Additionally, Luk et al. [33] showed that $\varepsilon_{p t}=\varepsilon_{\mathrm{r}}$ for an incompressible material. Then, using solution (15), equation (22) is reduced to:

$\dot{\varepsilon}_{p l}=-\frac{d v}{d r}=-\left(\frac{\dot{a}}{a}\right) \frac{d V}{d \xi}=2\left(\frac{\dot{a}}{a}\right) \frac{1}{\xi^{3}}$.

Replacing this solution in equation (2) in terms of $\xi$ and $\Sigma$, it is found:

$\Sigma=\Sigma_{0}\left(1+\left(\frac{2 \dot{a}}{a D} \frac{1}{\xi^{3}}\right)^{1 / q}\right)$

where $\Sigma_{0}=Y_{0} / E$ is the nondimensional yield stress at zero plastic strain. After that, replacing equation (24) in equation (16), a differential equation for $\Sigma_{\tau}$ is obtained:

$\frac{d \Sigma_{Y}}{d \xi}-\frac{2}{\xi} \Sigma_{0}\left(1+\left(\frac{2 a}{a D} \frac{1}{\xi^{3}}\right)^{1 / q}\right)=2 \dot{A}^{2}\left(\frac{\xi^{3}-1}{\xi^{5}}\right)$.

Equation (25) can be solved analytically using the boundary condition $\Sigma_{r}(\xi=1)=-p_{c} / E$ :

$$
\begin{aligned}
\Sigma_{r}(\xi)= & -\frac{p_{c}}{E}+2 \Sigma_{0} \ln (\xi)+\frac{3 \dot{A}^{2}}{2}+\frac{2 \dot{A}^{2}}{\xi}\left(\frac{1}{4 \xi^{3}}-1\right) \\
& +\frac{2 q}{3} \Sigma_{0}\left(\frac{2 \dot{a}}{a D}\right)^{1 / q}\left(1-\frac{1}{\xi^{3 / q}}\right),
\end{aligned}
$$

where $p_{c}$ is the pressure at the cavity wall.

The exact solution of equation (26) can be obtained numerically using equation (19) when $\xi=\xi_{i}$ as a boundary condition. However, an approximate solution was found using equations (20) and (21), and the elastic-plastic limit: $\Sigma\left(\xi=\xi_{i}\right)=\Sigma_{0}$. Therefore, it was found that $\xi_{i}^{3}=2 / 3 \Sigma$, and: 


$$
\frac{p_{c}}{E}=\frac{2}{3} \Sigma_{0}\left(1+\ln \left(\frac{2}{3 \Sigma_{0}}\right)\right)+\frac{3 \rho}{2 E} \dot{a}^{2}+\frac{2 q}{3} \Sigma_{0}\left(\frac{2}{a D}\right)^{1 / q}\left(1-\left(\frac{3 \Sigma_{0}}{2}\right)^{1 / q}\right) \dot{a}^{1 / q}
$$

This solution for cavity-expansion pressure (equation (27)) consists of a quasi-static term, an inertial term and a strain-rate term. Warren and Forrestal [34] obtained a similar analytical solution for a strain-rate-sensitive material. As was discussed in their paper, the strain-rate term includes the cavity radius $a$, which affects the assumption of the similarity transformation. However, a constant radius $a$ can be considered for penetration events that involve a sphericalnosed rigid penetrator (as the case of the current analysis). This is due to the fact that the nose of the projectile does not change its shape during the penetration process.

Finally, this solution can be simplified as:

$p_{c}=A_{\mathrm{s}}+C_{s} \frac{\rho}{2} \dot{a}^{2}+F_{s} \dot{a}^{1 / q}$

where $A_{s}$ is related with the quasi-static solution, because it does not depend on the velocity; $C_{s}$ is the inertial term, and $F_{s}$ is the strainrate-hardening contribution.

\subsubsection{Corrections at higher velocities}

Due to the fact that modeling clay has a low compression yield stress and a low modulus, it can be supposed that at a critical limit velocity $\left(v_{l}\right)$, the material can reach the fourth penetration regime (hyper-velocities), as was discussed in subsection 2.1. In this paper, this limit velocity was calculated using the upper limit of the hydrodynamic regime, as follows:

$v_{l}=\sqrt{\frac{K}{\rho}}$.

Then, it is necessary to correct the model (equation (28)) for velocities greater than $v_{l}$ in order to take into account hyper-velocities effects. One way to do this is to consider that clay begins to behave as a fluid; then, hydrodynamic conditions are achieved, and the Tate model can be applied $[20,21]$. Therefore, equation (28) reduces to:

$p_{c}=A_{\mathrm{s}}+\frac{\rho}{2} \dot{a}^{2}, \quad \dot{a}>v_{l}$.

Thus, at low velocities ( $\dot{a}<v_{l}$ ), equation (28) is applied to calculate $p_{c}$; otherwise, equation (30) is used. Notice that in equation (30), the strain-rate hardening term $\left(F_{s}\right)$ is no longer used because, at this point, the material no longer hardens with strain-rate changes (like a fluid).

\subsection{Engineering penetration model}

Fig. 2 shows a scheme of a spherical-nosed projectile penetrating a modeling clay target. In the figure, $a$ is the radius of the projectile with mass $m_{p}$; and $\phi$ and $x_{p}$ are the meridional angle and penetration depth on the clay, respectively. According to the second Newton's law, the movement of the projectile is governed by

$m_{p} \frac{d V_{p}}{d t}=m_{p} \frac{d^{2} x_{p}}{d t^{2}}=-F_{x}$

where $V_{p}$ is the velocity of the projectile, and $F_{x}$ is the force acting on its nose. The balance of forces on the cavity wall is given by $[35,36]$ :

$F_{x}=2 \pi \int_{0}^{x_{p}}\left(y y^{\prime} p_{c}+y f\right) d x$,

where $p_{c}$ is the pressure of the cavity acting on the nose, $f$ is the frictional forces (per unit of area) between the target and the

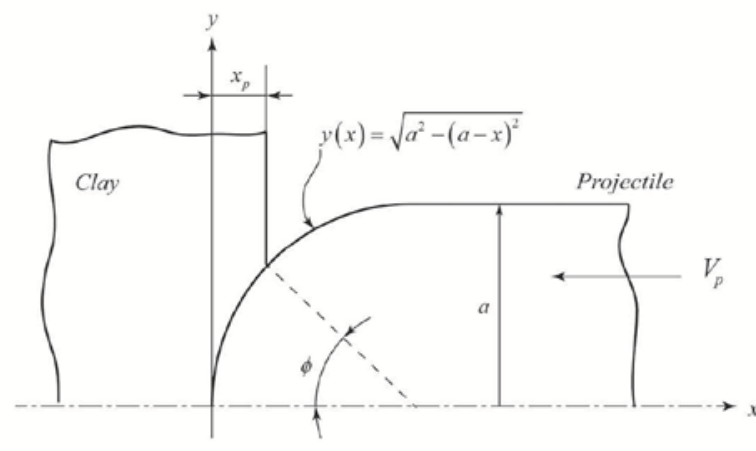

Fig. 2. Planar cross-section of a spherical-nosed projectile impacting a clay target.

projectile, and $y=y(x)$ is the shape function of an axis-symmetric penetrator with $0 \leq x \leq a$ [30]. Frictional force was assumed proportional to the radial stresses on the nose of the projectile (it means equal to the pressure on the cavity wall $p_{c}$ ) [37]; it is:

$f=\mu p_{c}$,

where $\mu$ is the dynamic sliding-friction coefficient. Therefore, replacing equation (33) and employing a hemispherical nose shape function $\left(y=\sqrt{a^{2}-(a-x)^{2}}\right)$ in equation (32), it is obtained:

$F_{x}=2 \pi \int_{0}^{x_{p}} p_{c}\left((a-x)+\mu \sqrt{a^{2}-(a-x)^{2}}\right) d x$.

In this equation, $p_{c}$ can be calculated using equation (28) at low velocities ( $\left.\dot{a}<v_{t}\right)$, and using equation (30) at high velocities $\left(\dot{a}>v_{t}\right)$.

The cavity-expansion velocity $(\dot{a})$ and the projectile velocity $\left(V_{p}\right)$ are related by (see Fig. 2) [38]:

$\dot{a}=V_{p} \cos \phi$,

where $\phi$ and $x_{p}$ are geometrically coupled by (see Fig. 2):

$\cos \phi=1-\frac{x_{p}}{a}$.

The penetration model is completed when $F_{x}$ (equation (34)) and all the previous involved relations are replaced in equation (31). Then, the position and velocity of the projectile can be found during the penetration event.

\section{Experimental procedure and results}

\subsection{Material}

The oil-based modeling clay used in this work is a commercially available soft Roma Plastilina No. 1 (RP) color gray-green provided by Sculpture House (USA). Prior to experimentation, the material was heated to $50{ }^{\circ} \mathrm{C}$ over 2 hours and manually homogenized to remove air gaps. After that, the material was held in a standard laboratory atmosphere (temperature $22 \pm 2{ }^{\circ} \mathrm{C}$; relative humidity $50 \pm 10 \%$ ) for over 88 hours.

The density of the material was determined with a displacement method in oil, and a value of $\rho=1529 \pm 5 \mathrm{~kg} / \mathrm{m}^{3}$ was obtained.

\subsection{Uniaxial compression tests}

Compression tests were carried out to characterize the RP at quasi-static (QS) conditions. They were performed following the recommendations made by Sofuoglu and Rasty [7] and Bermudez [39]. 


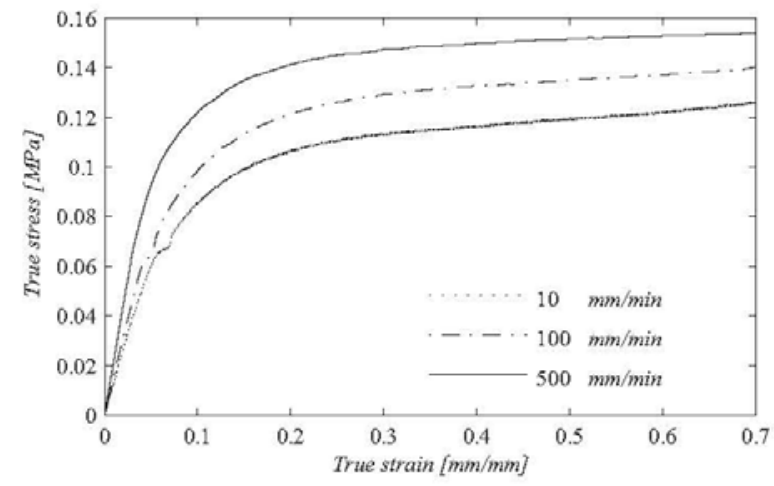

Fig. 3. Quasi-static true stress - true strain flow curves for Roma Plastilina No. 1 at three different compression speeds.

The specimens were in the form of a right circular cylinder of diameter $25.4 \mathrm{~mm}$ and $25.4 \mathrm{~mm}$ in height. An Instron Universal Testing Machine was used at three different cross-head speeds $\left(V_{c h}\right): 10$, 100 , and $500 \mathrm{~mm} / \mathrm{min}$, maintaining a constant temperature during the compression test at $22_{-10}^{+0.0}{ }^{\circ} \mathrm{C}$

Fig. 3 shows the flow curves for RP clay obtained using the standard compression test. The strain rate during the test can be calculated using the following relation [7]:

$$
\dot{\varepsilon}=\frac{V_{\text {ch }}}{H_{0} \exp (\varepsilon)},
$$

where $V_{c} h$ is the velocity of cross-head in the testing machine, $H_{0}$ is the initial length of the specimen and $\varepsilon$ is the true strain during the event. Notice in equation (37) that even though the $V_{c h}$ is constant during the compression test, the strain rate is changing. Therefore, a characteristic strain-rate is reported for the event. Thus, the strain-rates during the compression tests were $4.35 \times 10^{-3}$, $4.35 \times 10^{-2}$ and $2.17 \times 10^{-1} s^{-1}$ for the three $V_{c h}$ speeds, respectively.

The material parameters under OS conditions for the CS constitutive model were obtained from these quasi-static flow curves by curve fitting. They are summarized in Table 1. It is important to state that given the quasi-static state of the experiments and the small range of strain rates $\left(4.35 \times 10^{-3}\right.$ to $\left.2.17 \times 10^{-1} \mathrm{~s}^{-1}\right)$, the material parameters are not suitable for higher strain-rate ranges. Additionally, since the material parameters were determined independently, possible interactions among them are neglected.

\subsection{Coefficient of friction}

The coefficient of friction between the clay and the penetrator is an important parameter of the engineering model; for this reason, a methodology is proposed to measure this coefficient using the engineering penetration model at QS conditions. It is based on the quasi-static punch test, where a target of the RP clay is penetrated by a hemi-spherical punch, as can be seen in Fig. 4 .

The test was performed with the Instron Universal Testing Machine, with the punch fixed to the machine's cross-head, and

Table 1

Cowper-Symonds materials parameters of RP clay from quasi-static tests.

\begin{tabular}{llll}
\hline Elastic modulus & \multicolumn{4}{l}{ Cowper-Symonds parameters } \\
\hline$(E)$ & $\left(\mathrm{Y}_{0}\right)$ & $(D)$ & $(q)$ \\
$1.73 \mathrm{MPa}$ & $0.08 \mathrm{MPa}$ & $0.9192 \mathrm{~s}^{-1}$ & 9.034 \\
\hline
\end{tabular}

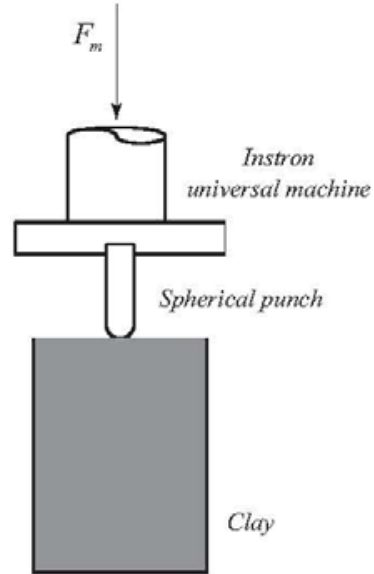

Fig. 4. Scheme of quasi-static punch test to determine coefficient of friction.

using a constant speed $V_{c h}$ of $10 \mathrm{~mm} / \mathrm{min}$. During the test, the force measured by the machine $\left(F_{m}\right)$ was recorded.

The force $F_{m}$ recorded by the Instron machine is presented in Fig. 5 (continuous light lines). Two different penetration tests (T1 and T2) were performed using the same punch conditions, as can be seen in the figure. $F_{m}$ was compared with the force calculated using the quasistatic part of the engineering model ( $A_{s}$ in the equation (28)) with the QS material properties of RP clay (Table 1), since the punch test was performed at constant and quasi-static penetration conditions.

The value of the coefficient of friction $\mu$ is fitted to obtain a good agreement between the measured and calculated force magnitude. Thus, the engineering model was numerically solved using different values of $\mu$, as shown in Fig. 5. Notice that when the extreme values of $\mu=0$ or $\mu=0.5$ are used (segmented lines), the force is not well predicted. A better prediction is achieved using a value of $\mu=0.149$ (continuous bold line). Therefore, for this study a value of the coefficient of friction between the materials was taken as 0.149 .

\subsection{Dynamic indentation test}

Based on the Tirupataiah and Sundararajan technique [40], a Dynamic Indentation (DI) test was performed as characterization

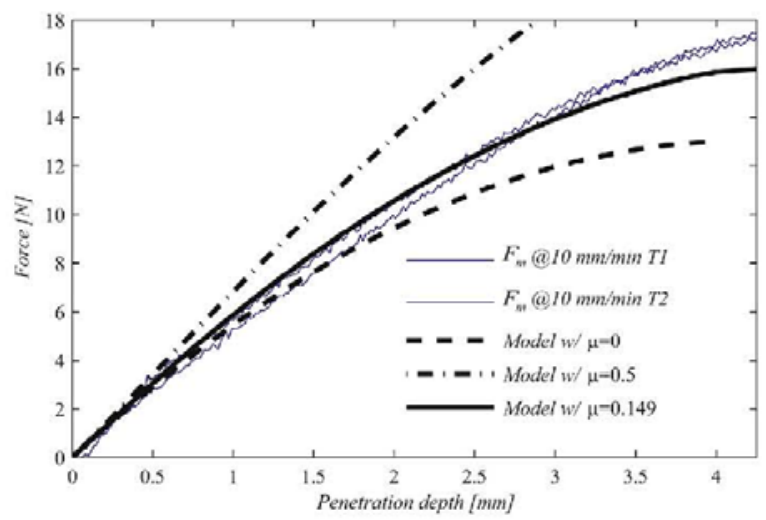

Fig. 5. Comparison between measured and calculated forces using different values of $\mu$. 


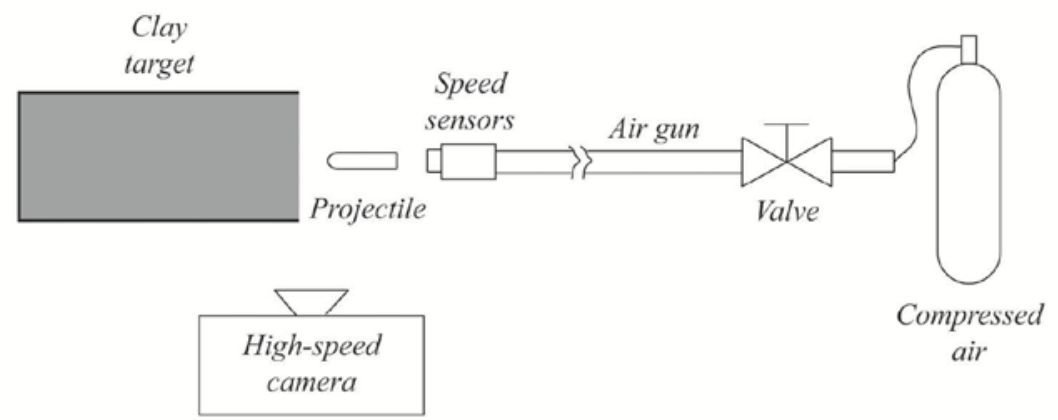

Fig. 6. Schematic view of dynamic indentation test setup.

of the modeling clay at high strain rates. In this test, a flat and semiinfinite test sample is impacted by a projectile at a range of impact velocities. For this purpose, an air gun with compressed air stored at the required pressure in a gas reservoir was used (see Fig. 6). A fast-acting solenoid valve was used to release the air into the gun. This gun is capable of propelling a $8.41 \mathrm{~mm}$ diameter, $6.62 \mathrm{~g}$, spherical-nosed, 6061-T4 aluminum projectile to velocities as high as $150 \mathrm{~m} / \mathrm{s}$. The velocity of the projectile could be altered by changing the air pressure. At the end of the gun, two photo-diode sensors were placed to measure the penetrator's initial velocity. The sensors were separated by a distance of $40 \mathrm{~mm}$ and connected to a digital timer. The timer measured the travel time of the projectile between the sensors; thus, the initial velocity of the projectile was calculated. Additionally, penetration was recorded with a high-speed digital camera, which is capable to capture up to 16 pictures during an event at high speed. Thus, penetration phenomena at different initial velocities were recorded with a speed in the range of 4000 to 7000 frames per second ( $f p s$ )

Before each DI test, the RP clay was conditioned on a rigid cylindrical container with diameter of $152 \mathrm{~mm}$ and length of $400 \mathrm{~mm}$. After that, the clay was prepared to obtain a smooth and even impact surface with respect to the container edges. This surface, flush with the edges of the container, was the reference level to measure the indentation depth.

The DI test was performed at four different initial penetration velocities: $20.84 \mathrm{~m} / \mathrm{s}, 32.03 \mathrm{~m} / \mathrm{s}, 40.84 \mathrm{~m} / \mathrm{s}$ and $43.44 \mathrm{~m} / \mathrm{s}$, which covered a characteristic strain-rate range from $2300 \mathrm{~s}^{-1}$ to $17900 \mathrm{~s}^{-1}$. Higher velocities could not be used because the projectile penetrates completely; in this case, it is impossible to acquire its position from the images. Thereby, only the final total penetration of the projectile into the clay was measured.

The characteristic strain-rates $\dot{\varepsilon}_{c h}$ in the test were calculated using the following equation [41]:

$\dot{\varepsilon}_{c h}=\frac{V_{0}}{2 a}$,

where $V_{0}$ is the initial penetration velocity, and $a$ is the projectile's radius.

The penetration sequence of the projectile at $V_{0}=40.84 \mathrm{~m} / \mathrm{s}$ penetrating the RP clay is shown Fig. 7; similar sequences were recorded for other initial velocities.

The images were scaled using the reference length scale in the photo. This scale was placed at the same focus and depth as the projectile to avoid scaling errors. With these images, the position of the projectile with time was extracted, following the rear of the projectile on each image. Time intervals between images were calculated using the camera's speed, and thus, the projectile's profile during its penetration on clay was obtained.

\subsection{Characterization method using genetic algorithms}

First, the complete engineering penetration model was programmed in Simulink-Matlab, using all the differential equations described in subsections 2.3 and 2.4. Therefore, it can be numerically solved, and the penetration depth can be found.

According to the proposed formulation, the calculated penetration depth $\left(x_{p}^{c}\right)$ can be expressed as function of geometrical $\left(m_{p}, a\right)$, material $\left(\rho, E, Y_{0}, D, q\right)$ and experimental parameters $\left(\mu, V_{p}\right)$ :

$x_{p}^{c}=f\left(m_{p}, a, \rho, E, Y_{o}, D, q, \mu, V_{p}\right)$,

where the geometrical and experimental parameters are known.

To identify the material constitutive parameters of the RP clay, an inverse analysis was implemented, where the difference between the experimental data from the DI test $\left(x_{p}^{m}\right)$, and mathematically calculated penetration results $\left(x_{p}^{c}\right)$ was minimized using the Euclidean metric $e\left(x_{p}\right)[42]$ :

$e\left(x_{p}\right)=\sqrt{\sum_{i=1}^{d}\left(x_{p}^{m}-x_{p}^{c}\right)^{2}}$,

where $d$ is the length of the array containing experimental data from a particular penetration test. Minimization of equation (40) was also implemented in the Matlab using the Genetic Algorithm Toolbox [43], and the configuration parameters are shown in Table 2.

After the minimization analysis, a new set of material parameters was determined. They were called dynamic parameters (D.P.) since they were extracted using the DI tests. Table 3 shows 10 runs of the Genetic Algorithm minimization analysis, showing repeatability of the optimization process. The DI parameters were taken as the average of the 10 runs.

\subsection{Finite-element model}

Explicit simulations were used to compare and verify the obtained results. Thus, the dynamical indentation test was implemented

Table 2

Parameters of GA toolbox for the minimization analysis [43].

\begin{tabular}{ll}
\hline Parameter & Value \\
\hline Initial population size & 50 \\
Creation function & Uniform \\
Scaling function & Proportional \\
Selection & Roulette \\
Reproduction & 1 elite count \\
Crossover fraction & 0.5 \\
Mutation & Uniform \\
Mutation rate & 0.2 \\
Crossover & Heuristic \\
Migration & Forward \\
\hline
\end{tabular}



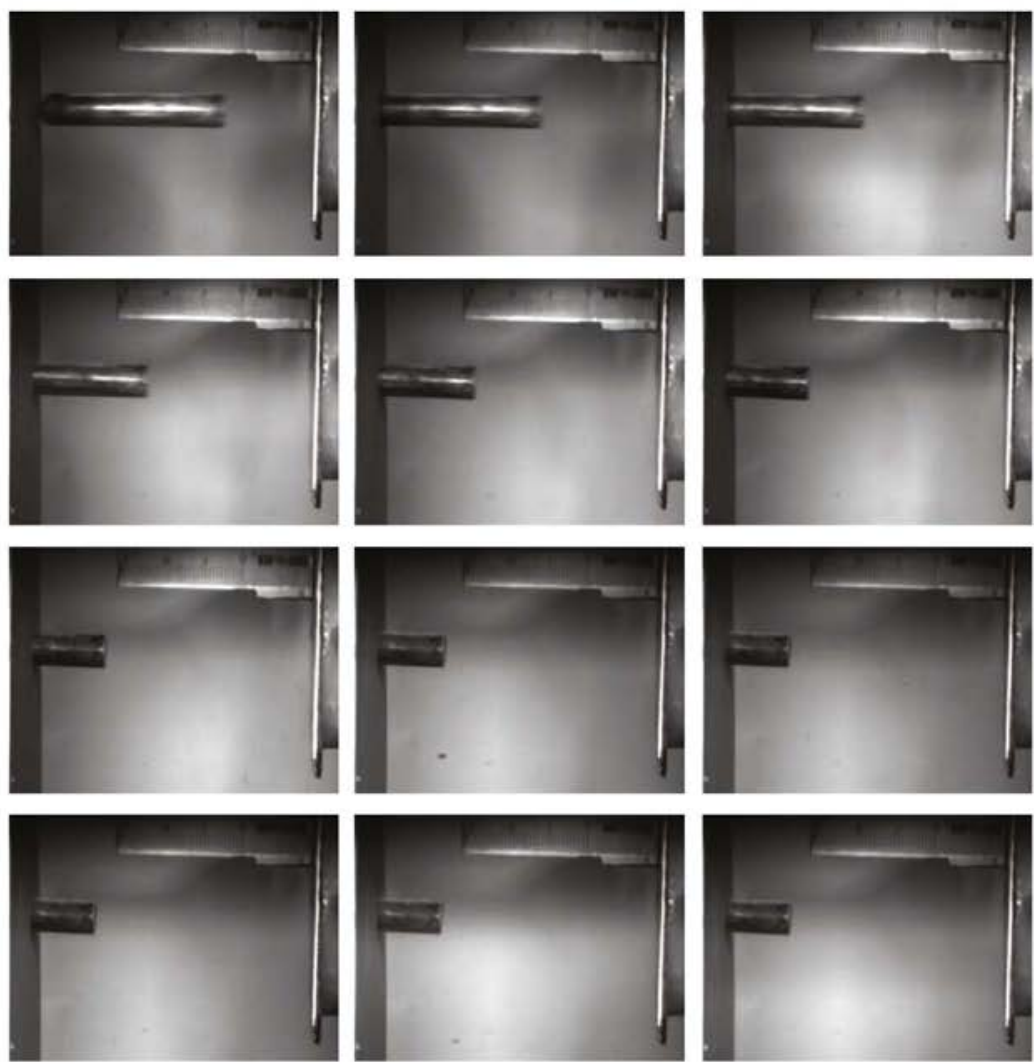

Fig. 7. High-speed sequence of penetration event at $40.84 \mathrm{~m} / \mathrm{s}$. Images taken at $5468 \mathrm{fps}$.

on ANSYS (AUTODYN) explicit software, using axial symmetry. The projectile was modeled as linear-elastic aluminum material (Table 4) and meshed reasonably fine to maintain a node spacing on the surface finer than on the surface of the modeling clay to promote a proper distribution of contact forces. Moreover, the RP clay was modeled as a mesh-free body using a Smoothed-Particle-Hydrodynamic(SPH) solver with a particle size of $0.2 \mathrm{~mm}$, and with the CowperSymonds Strength model and both set of material parameters: found by QS tests and found by the inverse problem. The initial velocity of the projectile $\left(V_{0}\right)$ was set equal to the velocity measured in the

Table 3

Dynamical parameters (D.P.) of Roma Plastilina No. 1 determined with DI test and inverse method.

\begin{tabular}{lllll}
\hline Run & $E[\mathrm{MPa}]$ & $\gamma_{0}[\mathrm{MPa}]$ & $D\left[\mathrm{~s}^{-1}\right]$ & $q_{[-]}$ \\
\hline 1 & 11.83 & 0.153 & 0.172 & 6.99 \\
2 & 11.89 & 0.153 & 0.197 & 7.00 \\
3 & 11.32 & 0.153 & 0.180 & 7.00 \\
4 & 11.42 & 0.153 & 0.184 & 6.99 \\
5 & 11.88 & 0.153 & 0.197 & 6.99 \\
6 & 11.47 & 0.153 & 0.174 & 7.00 \\
7 & 11.95 & 0.153 & 0.163 & 6.99 \\
8 & 10.93 & 0.152 & 0.181 & 6.97 \\
9 & 11.98 & 0.153 & 0.168 & 7.00 \\
10 & 11.70 & 0.153 & 0.199 & 7.00 \\
Mean & 11.64 & 0.153 & 0.181 & 6.99 \\
St. Dev. & 0.34 & $<0.001$ & 0.013 & $<0.01$ \\
Upper 95\% C.I. & 11.882 & 0.1531 & 0.1904 & 6.998 \\
Lower 95\% C.I. & 11.392 & 0.1527 & 0.1714 & 6.985 \\
\hline
\end{tabular}

C.., confidence interval. experimental test. Friction between the modeled bodies was configured using the value of $u=0.149$, as discussed in Section 3.3. Fig. 8 shows a sequence of the simulated process for the DI test, for the projectile velocity $V_{0}=40.84 \mathrm{~m} / \mathrm{s}$.

\subsection{Comparison berween quasi-static and dynamic parameters}

Figs. 9 and 10 show the comparison of the penetration depth predictions of the engineering model at different initial velocities for both set of parameters: using the quasi-static parameters (Q-S.P.) (dash-dot line), and using the dynamic parameters (D.P.) (solid line). Additionally, these figures also show the penetration depth results of the FE model using Q-S.P. (dotted line) and the D.P. (dashed line).

When the Q-S.P. are used, the model deviates sharply from the experimental data in the initial phase of the impact event, and the final penetration is over-predicted by both engineering and FE models. Thus, it is clear that these parameters are not suitable to represent the behavior of clay at high strain rates. On the other hand, the penetration is well predicted when the D.P. are used in the engineering and FE models and, hence, they can adequately predict the high-strain performance of the clay.

Table 4

Parameters of aluminum for simulation model [44].

\begin{tabular}{lll}
\hline Density & Elastic modulus & Poisson ratio \\
\hline$(\rho)$ & $(E)$ & $(v)$ \\
$2710 \mathrm{~kg} / \mathrm{m}^{3}$ & $69 \mathrm{GPa}$ & 0.33 \\
\hline
\end{tabular}



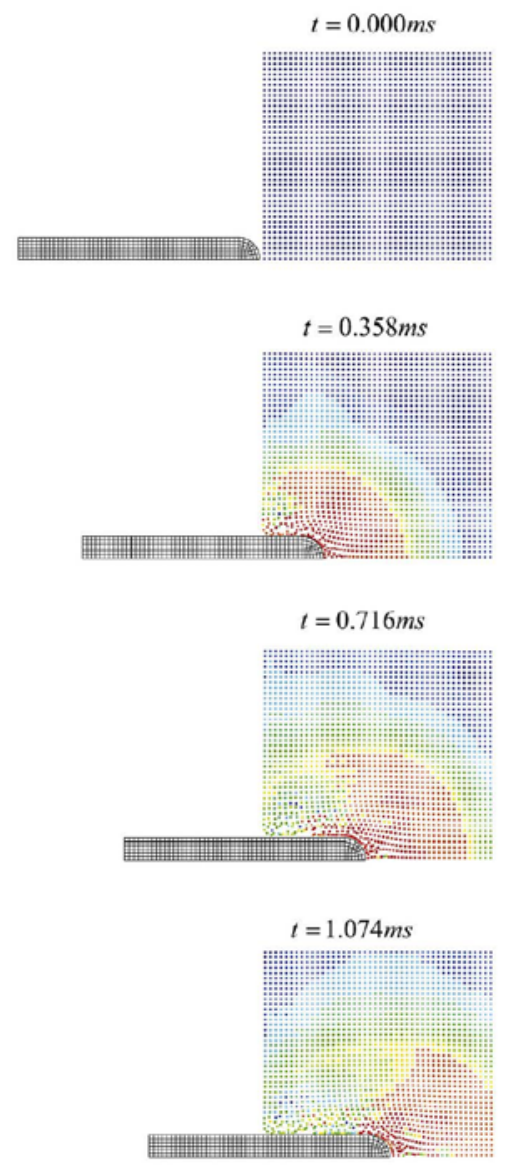
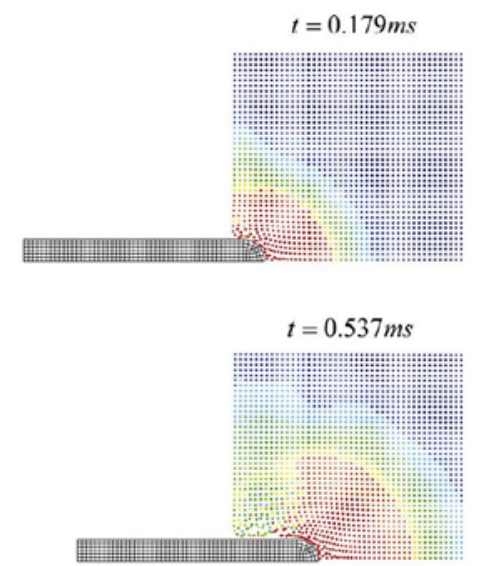

$t=0.895 \mathrm{~ms}$

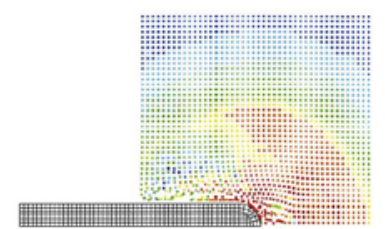

$t=1.254 \mathrm{~ms}$

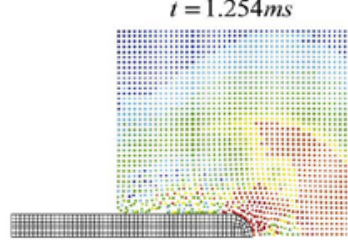

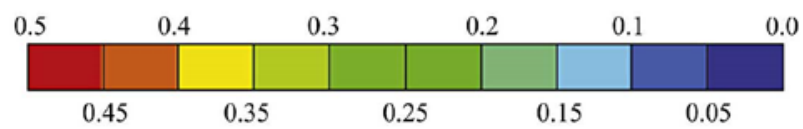

Fig. 8. Results of simulation sequence for DI test. Projectile velocity $40.84 \mathrm{~m} / \mathrm{s}$. Clay was modeled using the D.P. found with the optimization process (Table 3). The color scale represents Von-Mises stress (in MPa) in the clay.

\subsection{Results for higher velocities}

For higher velocities, only the final penetration depth was measured as was explained in Section 3.4. Fig. 11 compares predictions of the total penetration obtained by models with the experimental data. First, the penetration was calculated using the engineering model with the Q-S.P. (dash-dot line); the same over-prediction was found. After that, the engineering model with the D.P. and without the hyper-velocity correction was employed (solid red line); it means, equation (28) was only used to calculate the pressure on the cavity wall. Similar to the results shown in Figs. 9 and 10, there is a good agreement between the experimental data and the simulation results using D.P. at low velocities. However, velocities above $50 \mathrm{~m} / \mathrm{s}$, without the hyper-velocity correction, under-predicted the total penetration. This can be explained by the effect of hydrodynamic regime in the clay, which starts to behave as a fluid at high velocities. As a result, the material does not harden anymore, and only the inertial effects define the target's response. For this reason, the penetration model with rate-strain hardening is only valid up to this velocity level.

In contrast, the engineering model with the D.P. and with the hyper-velocity correction (solid black line) demonstrates a good agreement with the experimental results, as can be seen in the figure. It is remarked that the hyper-velocity correction was made using equations (28) and (30) to calculate the pressure on the cavity wall (as explained in subsection 2.3.3).

Results of some FE simulations using the D.P. are also shown in Fig. 11 (diamond markers). They are also in good agreement with the experimental points, validating suitability of the found D.P. for analysis of such high-rate behavior of the clay.

\section{Discussion}

In the formulation of the developed engineering model, there were some important points that must be further evaluated. 


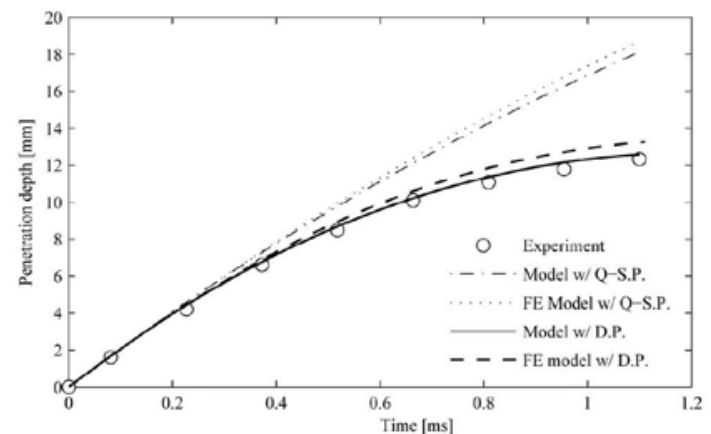

(a)

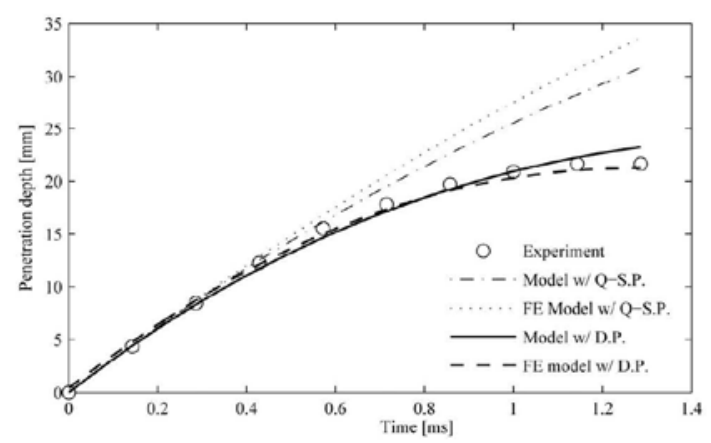

(b)

Fig. 9. Comparison with experimental data of the results obtained with engineer ing model and numerical simulations: (a) $20.85 \mathrm{~m} / \mathrm{s}$; (b) $32.03 \mathrm{~m} / \mathrm{s}$.

First, about the measured level of the coefficient of friction $\mu$. The technique proposed is valid for constant and quasi-static conditions; therefore, $\mu$ should be valid for this regime. However, the same value is used for high strain rates. Some researchers tried to calculate the dynamic coefficient of friction. Sofuoglu et al. [45] performed a ring-compression test for black plasticine, and using generic friction calibration curves, it was possible to find a value of $0.15<\mu<0.4$. However, Mandic and Stefanovic [46] argued those results since, according to them, generic frictional calibration curves cannot be used for all types of materials. Therefore, they constructed new frictional calibration curves for green plasticine. With those curves, the values of friction could be greater than previous ones. Obviously, they depend on the type of clay used by researchers. Another value $-\mu=0.15$ - was found by Andrade et al. [47] for three different types of clay, using a compression test and a cylindrical expansion model. Additionally, Aydin et al. [48] used a constant value of $\mu=0.2$ in an extrusion process with plasticine, and Ogasawara et al. [49] $\mu=0.15$ in an indentation process. With these references, and taking into account the difficulties of measurement, it is possible to assume the constant value found with the method described in this paper, since it does not differs much from the literature data.

Another important point is the use of the spherical cavityexpansion theory in the formulation. Some researchers showed that the use of this approach is more suitable to model long penetration events. However, a cylindrical penetration problem is adequate for sharp penetrators such as conical or ogival-nosed projectiles, because they force the radial displacement of material normal to the penetration direction. In our case, the use of spherical cavity expansion is more suitable due to the geometry of the nose, which

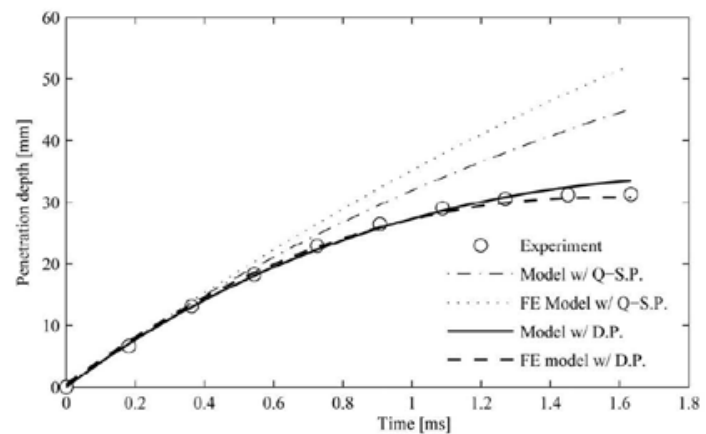

(a)

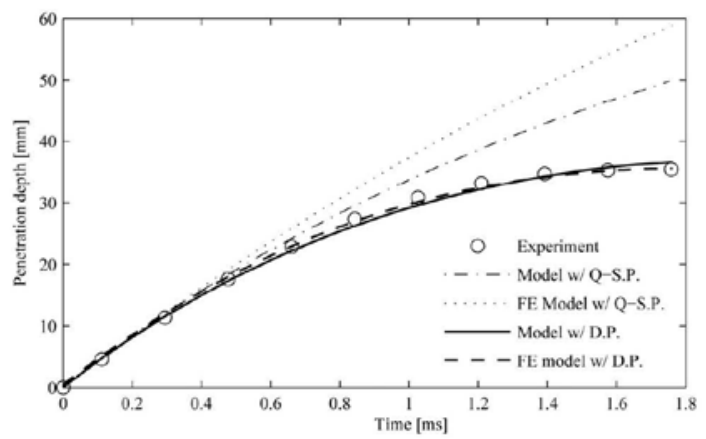

(b)

Fig. 10. Comparison with experimental data of the results obtained with engineer ing model and numerical simulations: (a) $40.84 \mathrm{~m} / \mathrm{s}$; (b) $43.44 \mathrm{~m} / \mathrm{s}$.

produces movements of spherical waves into the material. It is clearly shown in the results of simulations (see Fig. 8).

Based on the engineering model proposed, Teland and Moxnes [50] showed that it was impossible to find an analytical theory for a penetration process that covers a broad range of velocities. Still, he suggested that by using spherical dynamic models for the first part of the cratering phase, and a hydrodynamic theory with a decreased drag coefficient for the tunneling phase, it was possible to obtain a reliable modeling scheme. In this paper, the same methodology was proposed, and the model was divided into two; it was

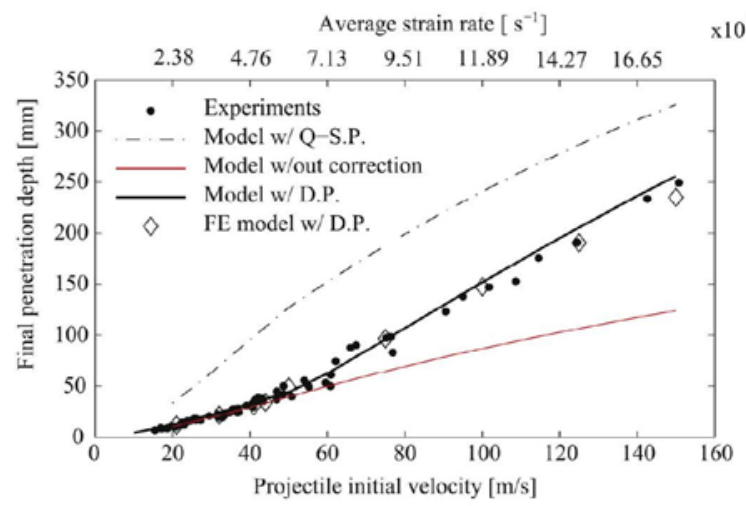

Fig. 11. Comparison of measured and predicted final penetration depth at differ ent initial velocities. 
justified by the description by Backman and Goldsmith [22], as explained in Section 2.1. Additionally, it is possible to calculate a value of $v_{l}$ using equation (29). Assuming the isotropic clay with $E=1.73 \mathrm{MPa}$ (Table 1) and $v=0.43$ [12], it was found that $v_{t}=51.45 \mathrm{~m} / \mathrm{s}$. This value correspond quite well with the transitional velocity found in experimental data and penetration models, as can be seen in Fig. 11.

Comparing the quasi-static parameters and the dynamic parameters (Tables 1 and 3 ), it can be noticed that they change drastically. First, elastic modulus $E$ increased from $1.73 \mathrm{MPa}$ to $11.64 \mathrm{MPa}$. Observing the tress-strain flow curves (Fig. 4), it was noticed that higher average strain-rate (velocity) led to higher $E$ values; even at this low range of strain rates. Additionally, Hernandez (hernandez2015) cal culated a value of $E=4.94 \mathrm{MPa}$ at medium strain rates $\left(10^{2} \mathrm{~s}^{-1}\right)$ as $4.94 \mathrm{MPa}$. Taking into account this tendency, higher $E$ values at higher strain rates $\left(<10^{3} \mathrm{~s}^{-1}\right)$ was expected. About the yield stress $\left(Y_{0}\right)$, it also increased from $0.08 \mathrm{MPa}$ to $0.153 \mathrm{MPa}$. Other researchers have found similar values; for example, Aydin et al. [48] calculated a value of $Y_{0}=0.11 \mathrm{MPa}$ using simulation of the extrusion process in plasticine; and Huang et al. [8] calculated a value of $Y_{0}=0.18 \mathrm{MPa}$ using indentation measurements on plasticine. Finally, related with the strain-rate parameters $D$ and $q$, it can be seen that they decreased, showing a reduction of the strain-rate effects at higher strainrates. This was also expected as strain-rate effects tend to disappear at hyper-velocities, as was mentioned in section 2.3.3.

Finally, due to the thixotropic behavior of this kind of soft materials, it is impossible to propose a general constitutive relation that can be applied to a wide range of loading conditions. Therefore, our formulation is only valid for the range of strain-rates studied and at constant temperature of $22^{\circ} \mathrm{C}$, as was demonstrated by the good agreement between the model and experimental results.

\section{Conclusions}

A methodology is proposed to characterize parameters of the Cowper-Symonds constitutive model for clay at high strain rates. This method employs a combination of a dynamic indentation testing, high-speed images and an engineering penetration model.

Using this methodology, the parameters of the Cowper-Symonds material model for Roma Plastilina at high strain rates were found, which agree reasonably well with simulations and experiments for a strain-range from $2300 \mathrm{~s}^{-1}$ to $17900 \mathrm{~s}^{-1}$.

\section{Acknowledgement}

The authors are grateful to the Industria Militar de Colombia (INDUMIL) for the availability of the high-speed camera used to obtain the experimental data presented in this paper.

\section{References}

[1] Committee on Testing of Body Armor Materials for Use by the U.S. Army. Testing of body armor materials: phase III. Ch 4. Clay and backing materials. Washington D.C. The National Academies Press; 2012.

[2] Office of Justice Programs, Ballistic resistance of body armor NIJ standard0101.06. National Institute of Justice (2008)

[3] Park JL, Chi YS. Hahn MH, Kang TJ. Kinetic dissipation in ballistic tests of soft body armors. Exp Mech 2012;52(8):1239-50.

[4] Hanlon E, Gillich P. Origin of the 44-mm behind-armor blunt trauma standard. Mil Med 2012;177(3):333-9.

15] Chijiliwa K. Hatamura Y. Hasegawa N. Characteristics of plasticine used in the simulation of slab in rolling and continuous casting. J Iron Steel Inst Jpn simulation of slab in

[6] Sofuoglu H. Physical modeling of extrusion process [Master's thesis]. Texas Tech University; 1990

[7] Sofuoglu H, Rasty J. Flow behavior of plasticine used in physical modeling of metal forming processes. Tribol Int 2000:33(8):523-9.

[8] Huang Z. Lucas M. Adams MJ. A numerical and experimental study of the indentation mechanics of plasticine. J Strain Anal Eng 2002;37(2):141-50.
[9] Eckerson K. Liechty B. Sorensen CD. Thermomechanical similarity between van aken plasticine and metals in hot-forming processes. J Strain Anal Eng 2008;43(5):383-94

[10] Hernandez C, Buchely MF, Maranon A. Dynamic characterization of Roma Plastilina No.1 from drop test and inverse analysis. Int J Mech Sci 2015; 100:158 68.

[11] Crandall S, Kurzweil L, Nigam A, Remington P. Dynamic properties of modelling clay. Tech. Rep., Acoustics and Vibration Laboratory, 1970.

[12] Crandall SH, Kurzweil LG, Nigam AK. On the measurement of Poissons ratio for modeling clay. Exp Mech 1971;11(9):402-13.

[13] Munusamy R, Barton DC. Behaviour of Roma Plastilina upon blunt projectile impact, in: DYMAT Conference, 2009, 749-55.

[14] Goodeve CF. A general theory of thixotropy and viscosity. T Faraday Soc $1939 ; 35: 342-58$

[15] Calvit H, Rader D, Melville J. Some wave-propagation experiments in plasteline. clay rods. Exp Mech 1968:8(9):418-23.

[16] Cowper GR. Symonds PS. Strain-hardening and strain-rate effects in the impact loading of cantilever beams, Tech. Rep., Brown University. Division of Applied Mathematics (1957).

[17] Nemat-Nasser S. Introduction to high strain rate testing, in: ASM Handbook Volume 8. Mechanical Testing and Evaluation. ASM International, 2000,

[18] Hill R. The mathematical theory of plasticity. Oxford: Oxford University Press: 1950.

[19] Hopkins HG. Dynamic expansion of spherical cavities in metals. Prog Solid Mech $1960 ; 1(3): 85-166$

[20] Tate A. A theory for the deceleration of long rods after impact. I Mech Phys Solids 1967; 15(6):387-99.

[21] Tate A. Further results in the theory of long rod penetration. J Mech Phys Solids 1969;17(3):141-50

[22] Backman ME, Goldsmith W. The mechanics of penetration of projectiles into targets. Int J Eng Sci 1978:16(1):1-99.

[23] Boutelier D, Schrank C, Cruden A. Power-law viscous materials for analogue experiments: New data on the rheology of highly-filled silicone polymers. J Struct Geol 2008:30:341-53.

[24] Heard H, Borg IC, Borg I, Carter N, Raleigh C. Flow and fracture of rocks, vol 16. Washington, DC: American Geophysical Union; 1972.

[25] Buchely MF, Maranon A. An engineering model for the penetration of a rigid-rod into a Cowper-Symonds low-strength material. Acta Mech 2015;226(9):2999 3010.

[26] Green AP. The use of plasticine models to simulate the plastic flow of metals. Philos Mag 1951:42(327):365-73.

[27] Feng WQ. Tong F. Tao XM. Experimental study on the behavior of a plasticine material, in: Bifurcation and Degradation of Geomaterials in the New Millennium. Springer, 2015. 293-8

[28] Durban D. Masri R. Dynamic spherical cavity expansion in a pressure sensitive elasto-plastic medium. Int J Solids Struct 2004;41(20):5697-716.

129] Forrestal MJ, Luk VK. Dynamic spherical cavity-expansion in a compressible elastic-plastic solid.J Appl Mech 1988:55:755-60.

[30] Masri R. Durban D. Dynamic spherical cavity expansion in an elasto-plastic compressible Mises solid. J Appl Mech 2005:72(6):887-98.

[31] Durban D. Baruch M. On the problem of a spherical cavity in an infinite elasto-plastic medium. J Appl Mech 1976:43(4):633-8.

[32] Durban D. Fleck NA. Spherical cavity expansion in a Drucker-Prager solid. ] Appl Mech 1997;64(4):743-50.

133] Luk VK, Forrestal M. Amos DE. Dynamic spherical cavity expansion of strainhardening materials. J Appl Mech 1991:58(1):1-6.

[34] Warren T, Forrestal M. Effects of strain hardening and strain-rate sensitivity on the penetration of aluminum targets with spherical-nosed rods. Int J Solids Struct 1998:35(28):3737-53.

[35] Jones S, Rule WK, Jerome D. Klug R. On the optimal nose geometry for a rigid penetrator. Comput Mech 1998;22(5):413-17.

[36] Jones S. Rule WK. On the optimal nose geometry for a rigid penetrator, induding the effects of pressure-dependent friction. Int J Impact Eng 2000;24(4):403-15.

37] Forrestal M, Okajima K, Luk VK. Penetration of 6061 -t651 aluminum targets with rigid long rods. J Appl Mech 1988;55:755-60.

[38] Forrestal M]. Tzou DY, Askari E, Longcope DB. Penetration into ductile metal targets with rigid spherical-nose rods. Int J Impact Eng 1995; 16(5-6):699-710.

[39] Bermudez R. Modelo experimental de plastilina para el estudio del modo de deformacion de materiales bajo indentacion puntiaguda [Master thesis]. Universidad Politecnica de Catalunya, Barcelona; 2011.

[40] Tirupataiah Y, Sundararajan G. A dynamic indentation technique for the characterization of the high strain rate plastic flow behaviour of ductile metals and alloys. J Mech Phys Solids 1991:39(2):243-71.

[41] Segletes SB. Modeling the penetration behavior of rigid spheres into ballistic gelatin, Tech. rep.., ARL-TR-4393(2008)

142] Cha S.H. Comprehensive survey on distance/similarity measures between probability density functions. Int ] Math Models Met AppISci 2007:1(4):300-7.

43) MathWorks, Genetic Algorithm and direct search toolbox for use with Matlab (2004).

[44] A. S. Handbook. Aluminum and aluminum alloys. Geauga County, OH: ASM International: 1993. p. 117

[45] Sofuoglu H, Gedikli H, Rasty ]. Determination of friction coefficient by employing the ring compression test. J Eng Mater Technol 2001:123(3):338-48.

[46] MandicV. Stefanovic M. Friction studies utilizing the ring compression test part $\mathrm{i}$, in: 8th International Tribology Conference, 2003, 46-51. 
[47] Andrade FAD, Al-Qureshi HA. Hotza D. Measuring and modeling the plasticity of clays. Mater Res 2010;13(3):395-9.

[48] Aydin L. Biglari FR, Briscoe B], Lawrence C], Adams MJ. Physical and numerical modelling of ram extrusion of paste materials: conical die entry case. Comp Mater Sci 2000:18(2):141-55.
[49] Ogas awara N. Chiba N. Chen X. A simple framework of spherical indentation for measuring elasto-plastic properties. Mech Mater 2009;41(9):1025-33.

[50] Teland JA. Moxnes JF. Analytical cavity expansion penetration models compared with numerical simulations, Tech. rep., Norwegian Defense Research Establishment (2003) 